\title{
Vitrificação de embriões equinos: alternativas para melhorar as taxas de sobrevivência embrionária
}

Vitrification of equine embryos: alternatives to improve embryonic survival rates

\author{
Andrez Pastorello Bohn'; Arnaldo Diniz Vieira'; Rafael Gianella Mondadori ${ }^{1}$ \\ ${ }^{1}$ Universidade Federal de Pelotas, Núcleo de Ensino e Pesquisa em Reprodução Animal (ReproPEL), Campus Capão \\ do Leão, RS, Brasil \\ Universidade Federal de Pelotas, Faculdade de Veterinária, Campus Universitário S/Nº, 96010-900, Capão do Leão,
} RS, Brasil.

\section{Resumo}

A criopreservação de embriões permite a criação de um banco genético de alto valor zootécnico, aproveitando-se do melhor momento reprodutivo das doadoras, facilitando a comercialização de material genético. Os crioprotetores utilizados durante o processo de vitrificação, afetam o equilíbrio osmótico, evitando a formação de cristais de gelo durante a diminuição de temperatura. Além da escolha adequada dos crioprotetores, o sistema de envase utilizado e a característica de manipulação prévia a vitrificação, impactam a viabilidade embrionária pós aquecimento. Apesar dos protocolos de vitrificação para embriões pequenos $(<300 \mu \mathrm{m})$ apresentarem taxas de prenhez aceitáveis, no momento de coletar essas estruturas da doadora a taxa de recuperação embrionária é baixa. Entretanto quando são avaliados os índices de sobrevivência de embriões grandes $(>300 \mu \mathrm{m})$, que apresentam maior taxa de recuperação, os mesmos protocolos não apresentam sucesso. A eficiência do processo de vitrificação em estruturas maiores está relacionada a redução do tamanho da blastocele, exigindo equipamentos de maior custo. $\mathrm{O}$ objetivo dessa revisão é descrever o "estado da arte" da vitrificação de embriões equinos, descrevendo diferentes protocolos de vitrificação, manipulação prévia e sistema de envase.

Palavras-chave: criopreservação, vitrificação de embriões, crioprotetores, biotecnologias, TE.

\section{Abstract}

Embryo cryopreservation allows the formation of a genetic bank from high genetical value animals, taking advantage of the best reproductive moment of the mares, facilitating its genetic material commercialization. Cryoprotectants used during the vitrification process act in osmotic equilibrium, preventing ice crystals formation during the temperature decrease. In addition to the appropriate choice of cryoprotectants, the characteristic of support/packaging used and the type of manipulation prior to vitrification impact the embryonic viability after heating. Although vitrification protocols for small embryos $(<300 \mu \mathrm{m})$ have acceptable pregnancy rates, their recovery rate is low. However, large embryos $(>300$ $\mu \mathrm{m})$ have higher recovery rates but low survival rates when the same protocols are used. Vitrification efficiency in larger structures is related to the reduction of blastocele size, requiring equipment with higher cost. The purpose of this review is to describe the state of the art of equine embryo vitrification by describing different vitrification protocols, previous manipulation and different storage systems.

Keywords: cryopreservation, embryo vitrification, cryoprotectant, biotechnolgy, ET.

\section{Introdução}

A criopreservação de embriões equinos em estágio de mórula compacta ou blastocisto inicial com diâmetro $<300 \mu \mathrm{m}$ permite a obtenção de boas taxas de prenhez (Slade et al., 1985; EldridgePanuska et al., 2005). Porém, estes estágios de desenvolvimento só são observados entre os dias 6 e 6,5 após a ovulação (D6 e 6,5), quando a taxa de embriões recuperados (D6 - 0\% e em D6,5 - 53\%) é inferior que no D7 (52\%) (Battut et al., 1997) e D8, onde o embrião apresenta diâmetro superior (Mccue et al., 2010). A menor taxa de recuperação se deve ao atraso no deslocamento do embrião pelo oviduto (Hinrichs e Choi, 2016). Assim sendo, adiar o momento da coleta representa uma alternativa para melhorar a taxa de recuperação.

Embriões equinos recuperados a partir do D7 geralmente possuem diâmetro $>300 \mu \mathrm{m}$, porém, já

${ }^{1}$ Correspondência: andrezbohn@gmail.com

Recebido: 06 de janeiro de 2020

Aceito: 18 de maio de 2020 
apresentam uma membrana glicoproteica (cápsula) no espaço perivitelino. Tais características dificultam o processo de criopreservação, que depende da substituição do conteúdo hídrico do embrião por crioprotetor, o que é dificultado pelo grande volume da blastocele e a baixa permeabilidade da cápsula (Scott et al., 2012). Desta forma, alternativas para reduzir o volume da blastocele dos embriões grandes e facilitar a permeabilidade do crioprotetor, tem demonstrado resultados promissores na melhora das taxas de criopreservação dessas estruturas (Choi et al., 2010; Scherzer et al., 2011; Diaz et al., 2016; Ferris et al., 2016; Weiss et al., 2016; Sanchez et al., 2017; Wilsher et al., 2018).

Dentre as estratégias utilizadas, estão a microperfuração por laser (Scherzer et al., 2011), micropunção manual (Ferris et al., 2016) ou assistida por micromanipulador (Choi et al., 2010; Diaz et al., 2016; Weiss et al., 2016; Sanchez et al., 2017; Wilsher et al., 2018). Nos trabalhos onde o colabamento da blastocele foi induzido, houve uma indicação de melhora na taxa de sobrevivência após a criopreservação por vitrificação, especialmente quando foi utilizada uma solução de vitrificação contendo dimetil sulfóxido (DMSO) e sistemas de envase abertos (Weiss et al., 2016; Sanchez et al., 2017; Wilsher et al., 2018). Desta forma, o objetivo deste trabalho é descrever o "estado da arte" da vitrificação de embriões equinos, descrevendo protocolos de vitrificação, manipulação prévia e sistemas de envase descritos na literatura.

\section{Vitrificação}

A vitrificação é um processo de criopreservação baseado na solidificação sem organização de cristais de gelo, atingindo um estágio amorfo com aspecto semelhante ao vidro. Essa condição pode ser obtida por diferentes vias, entretanto, para criopreservação mais eficiente é necessário a redução do volume das amostras e o uso de agentes crioprotetores para que seja possível atingir o estado vítreo. Nesse aspecto, os sistemas de envase (abertos ou fechados) para acondicionamento/armazenamento dos embriões podem afetar significativamente a velocidade de resfriamento, afetando a eficiência da vitrificação. Da mesma forma, a composição das soluções crioprotetoras afeta a capacidade de vitrificação e manutenção do estado vítreo, além de ter um importante papel no efeito tóxico sobre o embrião. Considerando o exposto, será feita uma descrição dos diferentes envases e soluções utilizadas na vitrificação de embriões equinos(Vajta e Nagy, 2006).

\section{Soluções de vitrificação - crioprotetores}

Assim como outros métodos de criopreservação, a vitrificação leva a um rearranjo da estrutura da membrana biológica, interferindo em sua funcionalidade (Holt, 2000). Os crioprotetores tem o objetivo de proteger os embriões dos efeitos críticos do processo. O mecanismo de proteção celular ocorre em parte, pela redução do ponto de solidificação das soluções durante o processo, entre outros fatores não totalmente elucidados (Fahy et al., 1987).

Os crioprotetores são solutos orgânicos que auxiliam na proteção de organelas, e uma das suas principais funções é remover e/ou substituir a água intracelular (Dobrinsky, 1996). Os agentes crioprotetores são utilizados para modular a formação de cristais de gelo quando se realiza a criopreservação, porém, a concentração do crioprotetor nos meios de criopreservação deve ser determinada pelo equilíbrio entre a prevenção da formação de cristais e a toxicidade da substância (Fahy, 1986; Fahy et al., 1987; Mclellan e Day, 1995). A toxicidade e o estresse osmótico são os principais causadores de danos as células (Papadopoulos et al., 2002) quando se utiliza altas concentrações de crioprotetores, por isso é importante a obtenção de altas taxas de resfriamento, reduzindo a toxicidade e fazendo com que o tempo de exposição do embrião as temperaturas próximas ao ponto de formação de cristais de gelo seja menor (Arav et al., 1993).

Os crioprotetores podem ser intra ou extracelulares (Fahy, 1987). Os mais utilizados para vitrificação de embriões equinos são o etilenoglicol (EG), glicerol (GLI) e o dimetil sulfóxido (DMSO), que são crioprotetores intracelulares. O EG e o GLI não possuem efeitos notavelmente tóxicos a membrana celular, sendo que o DMSO é o que requer maior atenção ao ser utilizado (Best, 2015). As suas ações protetoras são atribuídas as suas propriedades coligativas e ligantes com a água, diminuindo o ponto crioscópico intracelular (Holt, 2000). Temperaturas elevadas aumentam a velocidade de permeação dos crioprotetores, essa característica é bastante marcada para o DMSO, cuja hidrofobicidade aumenta com o aumento da temperatura, o que explica a crescente toxicidade do mesmo (Westh, 2004). Por sua vez, os crioproterores extracelulares atuam na proteção da membrana celular e por osmose, promovendo a 
Bohn et al. Vitrificação de embriões equinos: alternativas para melhorar as taxas de sobrevivência embrionária

desidratação celular controlada, sendo os principais representantes as macromoléculas de diferentes açúcares, como sacarose e glicose (Niemann, 1991).

\section{Sistema de acondicionamento do embrião}

\section{Palheta fechada}

A palheta fina $(0,25 \mathrm{~mL})$, é tradicionalmente utilizada no processo de congelamento, podendo também ser utilizada para vitrificação de embriões pequenos $(<300 \mu \mathrm{m})$. Por possuir grande volume, esse tipo de suporte limita a taxa de resfriamento a aproximadamente $2500^{\circ} \mathrm{C} /$ minuto quando é mergulhada no nitrogênio líquido, não sendo ideal para a vitrificação. Para aumentar a velocidade de resfriamento para aproximadamente $20.000^{\circ} \mathrm{C} /$ minuto, de modo a facilitar a vitrificação, deve ser reduzido o volume de meio ao redor do embrião (Carnevale, 2006; Stout, 2012).

\section{Open Pulled Straw (OPS)}

Método desenvolvido inicialmente para a vitrificação de oócitos e embriões bovinos (Vajta et al., 1998), onde a extremidade da palheta de $0,25 \mathrm{~mL}$ é aquecida e estirada, levando a uma diminuição de diâmetro. Por esse método as estruturas são envasadas em volumes de 1 ou $2 \mu \mathrm{L}$ de meio de vitrificação na extremidade afilada, que é mantida aberta. O método foi utilizado para vitrificação de embriões equinos recuperados D6-6,5 pós-ovulação, utilizando $2 \mu \mathrm{L}$ de soluções de vitrificação a base de DMSO e EG e sacarose. Nas avaliações in vitro, a vitrificação em OPS mostrou ser mais eficiente que a congelação convencional, obtendo-se uma menor taxa de embriões degenerados depois de três horas de cultivo, mas para confirmar a real eficiência da técnica deve-se transferir os embriões a éguas receptoras (Moussa et al., 2005).

\section{Cryoloop}

A primeira utilização do Cryoloop foi para a vitrificação de embriões de camundongo (Lane et al., 1999). A estrutura é composta por um círculo de nylon com espessura de $20 \mu \mathrm{m}$ e diâmetro entre 0,5 a $0,7 \mathrm{~mm}$. Em 2001 o suporte foi utilizado para a vitrificação de embriões equinos $<300 \mu \mathrm{m}$, com soluções a base de DMSO, EG e sacarose. Após vitrificação e aquecimento, durante cultivo in vitro, foi observado incremento na qualidade dos embriões (Oberstein et al., 2001).

\section{Hemi-palheta}

O sistema foi desenvolvido para a vitrificação de ovócitos e embriões humanos em estágio de blastocisto (Vanderzwalmen et al., 2000). A hemi-palheta é produzida manualmente mediante um corte longitudinal em uma palheta de $0,25 \mathrm{~mL}$, onde o embrião é depositado com um pequeno volume de solução de vitrificação. Esse sistema foi utilizado com embriões equinos no estágio de blastocisto expandido $(>300 \mu \mathrm{m})$ submetidos ao colabamento da blastocele. Com esse sistema, a transferência dos embriões vitrificados com solução a base de DMSO, EG e sacarose, resultou em $70 \%$ de prenhez (Sanchez et al., 2017).

\section{Cryolock}

O sistema Cryolock está disponível comercialmente e permite que a estrutura seja vitrificada com menos de $1 \mu \mathrm{L}$ de solução de vitrificação, demonstrando bons resultados em embriões equinos (Diaz et al., 2016). As soluções de vitrificação utilizadas foram a base de GLI e EG, seriadas em 3 concentrações e uma solução de diluição a base de galactose. É importante ressaltar que as estruturas criopreservadas possuíam diâmetro $>300 \mu \mathrm{m}$ e tiveram sua blastocele colapsada por aspiração com uso de micromanipulador. Os autores obtiveram cinco prenhezes de um total de seis embriões $(83 \%)$ submetidos a esse tratamento.

Cryoleaf

O Cryoleaf é um dispositivo para armazenamento e vitrificação de embriões e ovócitos, desenvolvido 
Bohn et al. Vitrificação de embriões equinos: alternativas para melhorar as taxas de sobrevivência embrionária

na Universidade de McGill, Montreal, Canadá. Ovócitos e embriões são preparados para a vitrificação de acordo com os protocolos de cada laboratório ou operador. Esse dispositivo proporciona vitrificação com baixo volume de meio, sendo a estrutura vitrificada em volume inferior a $1 \mu \mathrm{L}$ (Lopez et al., 2012).

O sistema foi utilizado para vitrificação de embriões equinos, com o colapso de blastocele assistido por laser. As soluções de vitrificação tiveram EG e DMSO, utilizadas em duas séries de concentrações. Os procedimentos resultaram em quatro prenhezes aos 11 dias, após a transferência de nove embriões (Scherzer et al., 2011).

As descrições demonstram que os diferentes sistemas de acondicionamento, com seus pontos positivos e limitações, já foram utilizados com relativo sucesso na vitrificação de embriões equinos.

\section{Métodos de colabamento de blastocele}

\section{Microperfuração por laser}

O método de microperfuração da blastocele por um sistema de laser é baseado em pulsos de laser direcionados a cápsula embrionária e a junção celular do trofectoderma. A abertura gerada pelos pulsos do laser permitem a saída do fluido da blastocele e a entrada da solução crioprotetora durante o processo de estabilização (Scherzer et al., 2007). Para a realização da perfuração, o embrião é posicionado com o embrioblasto na posição de nove horas e o laser penetra no embrião na posição de três horas (Scherzer et al., 2011).

A primeira utilização da microperfuração a laser foi descrita para embriões humanos, cujo objetivo foi melhorar as taxas de sobrevivência de blastocistos expandidos (Mukaida et al., 2006). Nesse trabalho, dos 40 embriões do grupo tratamento, foram obtidas 39 gestações $(97,5 \%)$. O indicativo de sucesso com embriões humanos, estimulou o utilização da técnica em embriões equinos com diâmetro $>300 \mu \mathrm{m}$, que foram posteriormente vitrificados com soluções de a base de DMSO e EG, utilizando o sistemas de acondicionamento CryoLeaf. O grupo tratamento resultou em quatro gestações aos 14 dias de um total de 11 embriões transferidos (Scherzer et al., 2011), segundo os autores, mais estudos tem que ser realizados para avaliar a criobriologia dos embriões equinos, visto que apenas uma receptora levou a gestação a termo.

\section{Micropunção com uso de micromanipulador}

Utilizando micromanipulador, diferentes métodos de micropunção realizados com diferentes ferramentas de manipulação embrionária já foram realizados. Os micromanipuladores usualmente utilizados para realizar a micropunção, são equipados com um braço para manter a posição do embrião com a pipeta (holding), e o outro braço no qual se acopla a agulha (diferentes diâmetros) para realizar o colapso da blastocele. Esses equipamentos foram utilizados em diversos trabalhos (Choi et al., 2010; Ferris et al., 2016; Weiss et al., 2016; Sanchez et al., 2017; Wilsher et al., 2018), sendo obtidas taxas de prenhez variáveis, o que estimula a realização de outros estudos.

Dentre os micromanipuladores o GeneSearch Embryo Cradle ${ }^{\circledR}$ é um equipamento que possui apenas um braço de manipulação, onde a pipeta que faz a microinjeção passa pelo interior da pipeta holding, responsável por manter a posição do embrião (Diaz et al., 2016). Esse equipamento também se mostrou eficiente no colabamento da blastocele de embriões equinos grandes antes da vitrificação, permitindo que a transferências dessas estruturas gerasse taxa de gestação de 83,3\%.

\section{Punção manual}

Esse método tem por objetivo gerar uma alternativa ao uso de equipamentos de elevado valor (micromanipulador). O procedimento consiste da realização do colapso da blastocele pela punção com agulha de $25 \mathrm{G}$ com controle manual. Os dados disponíveis mostram que a micromanipulação manual (prenhez de 46,7\%) não foi capaz de superar a micromanipulação assistida por equipamento (prenhez de $73 \%$ ) (Ferris et al., 2016).

\section{Considerações finais}

Conforme ficou evidenciado nos dados apresentados, embriões maiores (maior taxa de 
Bohn et al. Vitrificação de embriões equinos: alternativas para melhorar as taxas de sobrevivência embrionária

recuperação) não apresentam boas taxas de sobrevivência quando submetidos ao processo de criopreservação, assim sendo, o número de transferências de embriões equinos aumentou significativamente, sendo que o número de embriões criopreservados não acompanhou esse crescimento. Com o desenvolvimento de técnicas acessíveis, como o colapso manual da blastocele, acredita-se que o uso da crioproservação aumente, conforme demonstrado por Wilsher et al. (2018) que obtiveram taxas de prenhez satisfatórias com embriões criopreservados. O desenvolvimento das técnicas será fomentado pela necessidade do mercado, sendo esse um mercado disponível em todo mundo e ainda possui um vasto campo para pesquisa buscando melhorar os resultados, o que indica que a pesquisa de soluções para os problemas enfrentados atualmente seja brevemente contornado. Considerando o exposto se torna evidente as dificuldades em concluir qual é a melhor opção a ser escolhida e estabelecer um protocolo padrão, bem como, a necessidade de prosseguir com as atividades de pesquisa na área.

\section{Referências}

Arav A, Shehu D, Mattioli MJR. Osmotic and cytotoxic study of vitrification of immature bovine oocytes. J Reprod Fertil, v.99, n.2, p.353-358, 1993.

Battut I, Colchen S, Fieni F, Tainturier D, Bruyas JF. Success rates when attempting to nonsurgically collect equine embryos at 144, 156 or 168 hours after ovulation. Equine Vet J, n.25, p.60-2, 1997.

Best BP. Cryoprotectant Toxicity: Facts, Issues, and Questions. Rejuvenation Res, v.18, n.5, p.422-36, 2015.

Carnevale EM. Vitrification of equine embryos. Vet Clin North Am Equine Pract, v.22, n.3, p.831-41, 2006.

Choi YH, Gustafson-Seabury A, Velez IC, Hartman DL, Bliss S, Riera FL, Roldan JE, Chowdhary B, Hinrichs K. Viability of equine embryos after puncture of the capsule and biopsy for preimplantation genetic diagnosis. Reproduction, v.140, n.6, p.893-902, 2010.

Diaz F, Bondiolli K, Paccamonti D, Gentry GT. Cryopreservation of Day 8 equine embryos after blastocyst micromanipulation and vitrification. Theriogenology, v.85, n.5, p.894-903, 2016.

Dobrinsky JR. Cellular approach to cryopreservation of embryos. Theriogenology, v.45, n.1, p.17-26, 1996.

Eldridge-Panuska WD, Di Brienza VC, Seidel GE, Jr., Squires EL, Carnevale EM. Establishment of pregnancies after serial dilution or direct transfer by vitrified equine embryos. Theriogenology, v.63, n.5, p.1308-19, 2005.

Fahy GM. The relevance of cryoprotectant "toxicity" to cryobiology. Cryobiology, v.23, n.1, p.1-13, 1986.

Fahy GM, Levy DI, Ali SE. Some emerging principles underlying the physical properties, biological actions, and utility of vitrification solutions. Cryobiology, v.24, n.3, p.196-213, 1987.

Ferris RA, Mccue PM, Trundell DA, Morrissey JK, Barfield JP. Vitrification of large equine embryos following manual or micromanipulator-assisted blastocoele collapse. J Equine Vet Sci, v.41, p. 64-65, 2016.

Hinrichs K, Choi YH. Micromanipulation of equine blastocysts to allow vitrification. Rep Fertil Dev, 2016.

Holt WV. Basic aspects of frozen storage of semen. Anim Reprod Sci, v.62, n.1-3, p.3-22, 2000.

Lane M, Schoolcraft WB, Gardner DK. Vitrification of mouse and human blastocysts using a novel cryoloop container-less technique. Fertil Steril, v.72, n.6, p.1073-8, 1999.

Lopez E, Cipri K, Naso V. Technologies for Cryopreservation: Overview and Innovation. In: (Ed.). Current Frontiers in Cryobiology, 2012. cap. Chapter 11.

Mccue PM, Ferris RA, Lindholm A, Deluca C, Moyer W. Embryo recovery procedures and collection success: results of 492 embryo-flush attempts. Proceedings of the Annual Convention of the AAEP, 2010. p.318-321.

Mclellan MR, Day JG. Cryopreservation and freeze-drying protocols. Introduction. Methods Mol Biol, v.38, p.1-5, 1995.

Moussa M,Bersinger I,Doligez P,Guignot F,Duchamp G,Vidament M,Mermillod P, Bruyas JF. In vitro comparisons of two cryopreservation techniques for equine embryos: slow-cooling and open pulled straw (OPS) vitrification. Theriogenology, v.64, n.7, p.1619-32, 2005.

Mukaida T, Oka C, Goto T, Takahashi K. Artificial shrinkage of blastocoeles using either a microneedle or a laser pulse prior to the cooling steps of vitrification improves survival rate and pregnancy 
Bohn et al. Vitrificação de embriões equinos: alternativas para melhorar as taxas de sobrevivência embrionária

outcome of vitrified human blastocysts. Hum Reprod, v.21, n.12, p.3246-52, 2006.

Niemann HJT. Cryopreservation of ova and embryos from livestock: current status and research needs. Theriogenology, v.35, n.1, p.109-124, 1991.

Oberstein N, O'donovan MK, Bruemmer JE, Seidel GE, Jr., Carnevale EM, Squires EL. Cryopreservation of equine embryos by open pulled straw, cryoloop, or conventional slow cooling methods. Theriogenology, v.55, n.2, p.607-13, 2001.

Papadopoulos S, Rizos D, Duffy P, Wade M, Quinn K, Boland M, Lonergan PJaRS. Embryo survival and recipient pregnancy rates after transfer of fresh or vitrified, in vivo or in vitro produced ovine blastocysts. Anim Reprod Sci, v.74, n.1-2, p.35-44, 2002.

Sanchez R, Blanco M, Weiss J, Rosati I, Herrera C, Bollwein H, Burger D, Sieme H. Influence of Embryonic Size and Manipulation on Pregnancy Rates of Mares After Transfer of Cryopreserved Equine Embryos. J Equine Vet Sci, v.49, p.54-59, 2017.

Scherzer J, Davis C, Hurley DJ. Laser-assisted vitrification of large equine embryos. Reprod Domest Anim, v.46, n.6, p.1104-6, 2011.

Scherzer J, Fayrer-Hosken R, Ray L, Heusner GJR. A new approach to cryopreservation of large equine embryos by vitrification after blastocoel micromanipulation. Reprod Fertil Dev, v.19, n.1, p.225226, 2007.

Scott BR, Carwell DB, Hill RA, Bondioli KR,Godke RA, Gentry GT. Evaluation of Capsule Permeability in the Equine Blastocyst. J Equine Vet Sci, v.32, n.12, p.795-798, 2012.

Slade NP, Takeda T, Squires EL, Elsden RP, Seidel GE, Jr. A new procedure for the cryopreservation of equine embryos. Theriogenology, v.24, n.1, p.45-58, 1985.

Stout TA. Cryopreservation of equine embryos: current state-of-the-art. Reprod Domest Anim, v.47 Suppl 3, p.84-9, 2012.

Vajta G, Holm P, Kuwayama M, Booth PJ, Jacobsen H, Greve T, Callesen H. Open Pulled Straw (OPS) vitrification: a new way to reduce cryoinjuries of bovine ova and embryos. Mol Reprod Dev, v.51, n.1, p.53-8, 1998.

Vajta G, Nagy ZP. Are programmable freezers still needed in the embryo laboratory? Review on vitrification. Reprod Biomed Online, v.12, n.6, p.779-96, 2006.

Vanderzwalmen P, Bertin G, Debauche C, Standaart V, Schoysman E. Survival of Metaphase II Oocytes (MII) and Blastocysts After Vitrification in a Hemi-Straw (HS) System. Fertil Steril, v.74, n.3, p. S215-S216, 2000.

Weiss J, Blanco M, Sanchez R, Arbouin C, Schockemöhle P. Comparison between an open and a closed vitrification system for equine forced-collapsed embryos. J Equine Vet Sci, v.41, p.52-53, 2016.

Westh P. Preferential interaction of dimethyl sulfoxide and phosphatidyl choline membranes. Biochim Biophys Acta, v.1664, n.2, p.217-23, 2004.

Wilsher S, Rigali F, Couto G, Camargo S, Allen WR. Vitrification of equine expanded blastocysts following puncture with or without aspiration of the blastocoele fluid. Equine Vet J, 2018. 